\title{
Effect of Organic, Inorganic and Bio fertilizers on Soil Physicochemical Properties in Rainfed Maize-wheat Cropping System of Jammu
}

\author{
Bhoye Ranjanabai Chhagan, M.P. Sharma, K.R. Sharma, Abhijit Samanta, \\ Owais Ali Wani*, Dileep Kachroo, Manish Kumar, V.K. Razdan, \\ Vikas Sharma, A.K. Mondal and V.M. Arya
}
Division of Soil Science and Agricultural Chemistry, Sher-e-Kashmir University of Agricultural Sciences and Technology, Chatha, Jammu- 180009 (J\&K), India

*Corresponding author

\begin{abstract}
A B S T R A C T
\section{Keywords}

Organic, Inorganic Bio fertilizers, Soil Physicochemical Properties

Article Info

Accepted:

17 March 2019

Available Online:

10 April 2019

\section{Introduction}

Maize-wheat system in the sub-mountainous western Himalayan region of India comprises the state of Jammu and Kashmir. The contribution of this cropping system to total food grain production of the country is considerably large, amounting to $36.49 \%$ of wheat (93.90 million tonnes) and $8.38 \%$ of maize (21.57 million tonnes) which makes it one of the predominant cropping systems in India (Ramesh et al., 2014). However, in spite of the significant achievement made in research and development productivity of
\end{abstract}

An experiment was conducted during 2015-2016 in sandy loams at Jammu under the INM in maize-wheat system. The results revealed that the application of recommended levels of NPK to maize-wheat with FYM, VC and biofertilizers (Azotobacter and phosphate solubilizing bacteria) resulted in grain 66.53 per cent and straw 13.00 per cent increase over control in maize and wheat yields, respectively. Increasing levels of $50 \%$ $\mathrm{N}+$ recom. $\mathrm{P}+\mathrm{K}+\mathrm{S}+\mathrm{Zn}+\mathrm{B}+\mathrm{VC}+\mathrm{Azoto}+\mathrm{PSB}$ significantly increased the yield of both the crops. Integrated use of organic, inorganic and biofertilizers improved the soil status of available N, P, K, S over the initial values. A declining trend $(70.05,9.14,110.13,8.14 \mathrm{~kg}$ $\mathrm{ha}^{-1}$ ) from the initial value of available nutrients indicates a considerable mining of INM from the soil and suggest the need to adopt judicious organic, inorganic and biofertilizers.
Maize-wheat system is still very low. Maizewheat is dominant cropping sequence covering 2, 21,773 and $2,88,842$ ha area, respectively under rainfed condition of Jammu region. The average productivity of this system is being 3.44 tonnes/ha which is quite low as compared to national average of 5.6 tonnes/ha. It may be due to little use of organics, poor soil fertility because of coarse texture, and low organic matter content, heavy requirement of nutrients by these crops. Out of these, imbalanced application of nutrients is a major factor affecting crop productivity and soil characteristics in the 
region. In the present day of intensive agriculture, the crop plant is unable to use all the applied nutrients in its short lifespan. Therefore, fertilizer scheduling should be done on the bases of cropping sequence rather than individual crop to utilize residual plant nutrients for their efficient, economical and judicious use

The occurrence of deficiency of secondary nutrient viz., $\mathrm{S}$ and micronutrients viz., $\mathrm{Zn}$ and $\mathrm{B}$ is being observed increasingly under heavy feeder maize-wheat cropping sequence. Balanced fertilization to any crop or cropping sequence played vital role to attain optimum crop yield, enhance crop quality, corrects inherent soil nutrient deficiencies, sustain soil fertility, improve nutrient and water use efficiency, avoid damage to the environment and restore fertility of land that has been degraded by wrong and exploitative practices in the past. Adoption of such practices may influence carbon sequestration in soil because of their progressive effect on crop growth. Balanced nutrient management on the bases of soil testing can be achieved in better way by adopting integrated application of organic and inorganic for enhancing soil quality, input use efficiency and crop productivity which is the all most important for food and nutrient security. Maize-wheat is one of the most important double cropping systems being practiced under rainfed condition of Jammu region. Both, being exhaustive crops, required a huge amount of nutrients for producing higher yield. The best option in respect to balanced application of nutrients through organic and inorganic may come out for making the judicious fertilizer recommendations for realizing higher inherent yield potentials of both the crops as well as the outcome of this investigation will facilitate the stakeholders to maximize crop yield, nutrient use efficiency, water use efficiency, and soil quality under rainfed conditions of Jammu region.

\section{Materials and Methods}

The present investigation is a part of an ongoing experiment with maize-wheat cropping system in progress since kharif and rabi 2015 and 2016 at Advance center for rainfed Agriculture Rakh Dhainsar, SKUAST-Jammu and Department of Soil Science and Agricultural Chemistry, Chatha, Jammu Jammu and Kashmir ( $32^{\circ} 39^{\prime}$ N $\mathrm{N}$ and $\left.74^{0} 58 \mathrm{E}\right)$. Dhainsar, Jammu has a sub-tropical climate with a characteristic feature dry and cold winter. In winter season i.e Oct to March the temperature 2 to $200 \mathrm{c}$ and the relative humidity 41 to $65 \%$.Gernerally, dry and warm weather prevails during the months of March to June. The temperature in the month of May reaches as high as 48 0C. Monsoon season extends from first of July to mid-September. Total annual rainfall varies from $1049.2 \mathrm{~mm}$ to $1304 \mathrm{~mm}$ with the mean value of around $\mathrm{mm}$. The length of growing period of both the crops ranges from 200 to 210 days. The soil of the experimental field is sandy loam in texture having $\mathrm{pH}$ (6.67), Bulk density (1.64 $\mathrm{Mg} \mathrm{\textrm {m } ^ { - 3 }}$ ), Water holding capacity(11.01\%), organic carbon $\left(2.05 \mathrm{~g} \mathrm{~kg}^{-1}\right)$ and available nitrogen $\left(70.05 \mathrm{~kg} \mathrm{ha}^{-1}\right)$, Phosphorus $(9.14 \mathrm{~kg}$ $\left.\mathrm{ha}^{-1}\right)$, Potassium (110.13 $\left.\mathrm{kg} \mathrm{ha}{ }^{-1}\right)$ were, Sulphur $\left(8.14 \mathrm{~kg} \mathrm{ha}^{-1}\right)$ and Zinc $(0.36 \mathrm{mg}$ $\left.\mathrm{kg}^{-1}\right)$, Boron $\left(0.34 \mathrm{mg} \mathrm{kg}{ }^{-1}\right)$, respectively. There were eleven treatments viz., $\mathrm{T}_{1}$ Control, $\mathrm{T}_{2}$ Recommended NPK $\mathrm{T}_{3}$ Reco. NPK+ $\mathrm{S}+$ $\mathrm{Zn}+\mathrm{B}, \mathrm{T}_{4} 50 \% \mathrm{~N}+$ Reco. $\mathrm{P}+\mathrm{K}+\mathrm{S}+\mathrm{Zn}+$ $\mathrm{B}+50 \% \mathrm{~N}$ through FYM, $\mathrm{T}_{5} 50 \% \mathrm{~N}+$ Reco. $\mathrm{P}+\mathrm{K}+\mathrm{S}+\mathrm{Zn}+\mathrm{B}+50 \% \mathrm{~N}$ through $\mathrm{VC}, \mathrm{T}_{6}$ $75 \% \mathrm{~N}+$ Reco. $\mathrm{P}+\mathrm{K}+\mathrm{S}+\mathrm{Zn}+\mathrm{B}+25 \% \mathrm{~N}$ through FYM, $\mathrm{T}_{7} 75 \% \mathrm{~N}+$ Reco. $\mathrm{P}+\mathrm{K}+\mathrm{S}$ $+\mathrm{Zn}+\mathrm{B}+25 \% \mathrm{~N}$ through $\mathrm{VC}, \mathrm{T}_{8} 50 \% \mathrm{~N}+$ Reco. $\mathrm{P}+\mathrm{K}+\mathrm{S}+\mathrm{Zn}+\mathrm{B}+50 \% \mathrm{~N}$ through $\mathrm{FYM}+$ Azot +PSB, T9 50\% N + Reco. $\mathrm{P}+\mathrm{K}$ $+\mathrm{S}+\mathrm{Zn}+\mathrm{B}+50 \% \mathrm{~N}$ through $\mathrm{VC}+\mathrm{Azot}+$ PSB, $\mathrm{T}_{10} 75 \% \mathrm{~N}+\mathrm{Reco} . \mathrm{P}+\mathrm{K}+\mathrm{S}+\mathrm{Zn}+\mathrm{B}+25 \%$ $\mathrm{N}$ through $\mathrm{FYM}+$ Azto $+\mathrm{PSB}, \mathrm{T}_{11} 75 \% \mathrm{~N}+$ Reco. $\mathrm{P}+\mathrm{K}+\mathrm{S}, \mathrm{Zn}+\mathrm{B}+25 \% \mathrm{~N}$ through $\mathrm{VC}$ + Azot + PSB. Each treatment was replicated 
thrice in a randomized block design. The recommended $\mathrm{P}, \mathrm{K}, \mathrm{S}, \mathrm{Zn}$ and $\mathrm{B}$ doses, based on initial soil test, maize and wheat. The sources of $\mathrm{N}$ through FYM and VC were incorporated treatment. (Sowing time) in the soil sowing time of maize and wheat during kharif and rabi season since 2015-16. Treatments $\mathrm{T}_{8}, \mathrm{~T}_{9}, \mathrm{~T}_{10}$ and $\mathrm{T}_{11}$ involved inoculation by Azotobacter and PSB culture (@ $5 \mathrm{~g} \mathrm{~kg}^{-1}$ seed). Maize- wheat varieties used were Vivak Maize-25 and PBW-175 respectively, maize in general was sown on the set of monsoon (third week of July) as rainfed crop during kharif and wheat in the to fourth week of October as irrigation crop during rabi. The maturity and yield data were recorded after harvested at maturity and yield data were recorded after threshing. Soil samples from $0-15 \mathrm{~cm}$ depth were collected after harvest of maize in the 2 th cropping year (2015-16 and 2017) and were analyzed for available $\mathrm{N}$ by alkaline permanganate method (Subbiah and Asija, 1956), available P (Olsen et al., 1954) and available $\mathrm{K}$ (ammonium acetate extract). All observations were recorded for both the crops and soil properties were analyzed statistically

\section{Results and Discussion}

The highest magnitude of BD after harvesting of maize and wheat was recorded in $\mathrm{T}_{1}(1.54)$ to lowest in $\mathrm{T}_{6}(1.41)$ and $\mathrm{T}_{1}(1.52)$ to lowest in $\mathrm{T}_{6}(1.40)$. The highest $\mathrm{BD}$ may be due to high sand content and poor organic matter and clay content whereas lowest $\mathrm{BD}$ in $\mathrm{T}_{6}(1.41)$ could be due to addition of organic carbon and significant improvements of addition of organic, inorganic and biofertilizers which collectively influenced the BD in soils (Table $1-4)$.

Table.1 Integrated effect of inorganic, organic and biofertilizers on bulk density $\left(\mathrm{mg} \mathrm{m}^{-3}\right)$ of soil after harvesting maize wheat cropping system

\begin{tabular}{|c|c|c|c|c|c|c|c|}
\hline \multirow[b]{2}{*}{ Sr.No } & \multirow[b]{2}{*}{ Treatments } & $I^{\text {st }}$ year & II $^{\text {nd }}$ year & Overall & $I^{\text {st }}$ year & II $^{\text {nd }}$ year & Overall \\
\hline & & \multicolumn{3}{|c|}{$\left(\mathrm{mg} \mathrm{m}^{-3}\right)$} & \multicolumn{3}{|c|}{ (mg m-3) } \\
\hline T1 & Control & 1.54 & 1.52 & 1.53 & 1.51 & 1.53 & 1.52 \\
\hline $\mathbf{T 2}$ & Recommended NPK & 1.50 & 1.52 & 1.51 & 1.50 & 1.51 & 1.51 \\
\hline T3 & Reco. $\mathbf{N P K}+\mathrm{S}+\mathrm{Zn}+\mathrm{B}$ & 1.49 & 1.50 & 1.50 & 1.47 & 1.46 & 1.47 \\
\hline $\mathbf{T 4}$ & $\begin{array}{l}50 \% \mathrm{~N}+\operatorname{Reco.} \mathrm{P}+\mathrm{K}+\mathrm{S}+\mathrm{Zn}+\mathrm{B}+ \\
\mathbf{5 0 \%} \mathrm{N} \text { through FYM }\end{array}$ & 1.46 & 1.45 & 1.46 & 1.42 & 1.45 & 1.44 \\
\hline T5 & $\begin{array}{l}50 \% \mathrm{~N}+\operatorname{Reco.} \mathbf{P}+\mathrm{K}+\mathrm{S}+\mathrm{Zn}+\mathrm{B}+ \\
50 \% \mathrm{~N} \text { through } \mathrm{VC}\end{array}$ & 1.48 & 1.47 & 1.48 & 1.40 & 1.41 & 1.41 \\
\hline T6 & $\begin{array}{l}75 \% \mathrm{~N}+\operatorname{Reco.} \mathrm{P}+\mathrm{K}+\mathrm{S}+\mathrm{Zn}+\mathrm{B}+25 \% \\
\mathrm{~N} \text { through FYM }\end{array}$ & 1.41 & 1.38 & 1.40 & 1.46 & 1.47 & 1.47 \\
\hline T7 & $\begin{array}{l}75 \% \mathrm{~N}+\text { Reco. } \mathrm{P}+\mathrm{K}+\mathrm{S}+\mathrm{Zn}+\mathrm{B}+25 \% \\
\mathrm{~N} \text { through VC }\end{array}$ & 1.45 & 1.44 & 1.45 & 1.47 & 1.46 & 1.46 \\
\hline T8 & $\begin{array}{l}50 \% \mathrm{~N}+\text { Reco. } \mathrm{P}+\mathrm{K}+\mathrm{S}+\mathrm{Zn}+\mathrm{B}+ \\
50 \% \mathrm{~N} \text { through } \mathrm{FYM}+\mathrm{Azot}+\mathrm{PSB}\end{array}$ & 1.44 & 1.40 & 1.42 & 1.45 & 1.43 & 1.44 \\
\hline T9 & $\begin{array}{l}50 \% \mathrm{~N}+\operatorname{Reco.} \mathrm{P}+\mathrm{K}+\mathrm{S}+\mathrm{Zn}+\mathrm{B}+\mathbf{5 0 \%} \\
\mathrm{N} \text { through VC }+ \text { Azot }+\mathrm{PSB}\end{array}$ & 1.46 & 1.42 & 1.44 & 1.41 & 1.42 & 1.41 \\
\hline T10 & $\begin{array}{l}75 \% \mathrm{~N}+\text { Reco.P }+\mathrm{K}+\mathrm{S}+\mathrm{Zn}+\mathrm{B}+25 \% \mathrm{~N} \\
\text { through FYM }+ \text { Azto }+\mathrm{PSB}\end{array}$ & 1.43 & 1.40 & 1.42 & 1.42 & 1.41 & 1.41 \\
\hline T11 & $\begin{array}{l}75 \% \mathrm{~N}+\operatorname{Reco.} \mathrm{P}+\mathrm{K}+\mathrm{S}, \mathrm{Zn}+\mathrm{B}+25 \% \\
\mathrm{~N} \text { through } \mathrm{VC}+\mathrm{Azot}+\mathrm{PSB}\end{array}$ & 1.46 & 1.40 & 1.43 & 0.022 & 0.011 & 0.008 \\
\hline & \pm S.E.(m) & 0.003 & 0.014 & 0.012 & 0.064 & 0.033 & 0.025 \\
\hline & C.D. $(P=0.05)$ & 0.010 & 0.042 & 0.037 & 1.51 & 1.53 & 1.52 \\
\hline
\end{tabular}


Table.2 Integrated effect of inorganic, organic and biofertilizers on water holding capacity (\%) soil after harvesting maize wheat cropping system

\begin{tabular}{|c|c|c|c|c|c|c|c|}
\hline \multirow[t]{2}{*}{9.5} & \multirow[b]{2}{*}{ Treatments } & $I^{\text {st }}$ year & II $^{\text {nd }}$ year & Overall & $I^{\text {st }}$ year & II $^{\text {nd }}$ year & Overall \\
\hline & & \multicolumn{3}{|l|}{$(\%)$} & \multicolumn{3}{|c|}{$(\%)$} \\
\hline T1 & Control & 11.25 & 11.29 & 11.27 & 11.11 & 11.19 & 11.15 \\
\hline T2 & Recommended NPK & 11.34 & 11.43 & 11.39 & 11.15 & 11.33 & 11.24 \\
\hline T3 & Reco. $\mathrm{NPK}+\mathrm{S}+\mathrm{Zn}+\mathrm{B}$ & 11.37 & 11.94 & 11.66 & 11.19 & 11.63 & 11.41 \\
\hline T4 & $\begin{array}{l}50 \% \mathrm{~N}+\text { Reco. } P+K+S+Z n+B+50 \% \\
N \text { through FYM }\end{array}$ & 12.51 & 13.32 & 12.92 & 12.20 & 12.65 & 12.43 \\
\hline T5 & $\begin{array}{l}50 \% \mathrm{~N}+\text { Reco. } P+K+S+Z n+B+50 \% \\
N \text { through VC }\end{array}$ & 11.34 & 12.41 & 11.88 & 11.23 & 11.26 & 11.25 \\
\hline T6 & $\begin{array}{l}75 \% \mathrm{~N}+\text { Reco. } \mathrm{P}+\mathrm{K}+\mathrm{S}+\mathrm{Zn}+\mathrm{B}+25 \% \mathrm{~N} \\
\text { through FYM }\end{array}$ & 11.56 & 11.39 & 11.48 & 11.19 & 11.30 & 11.24 \\
\hline T7 & $\begin{array}{l}75 \% \mathrm{~N}+\text { Reco. } \mathrm{P}+\mathrm{K}+\mathrm{S}+\mathrm{Zn}+\mathrm{B}+25 \% \mathrm{~N} \\
\text { through VC }\end{array}$ & 11.27 & 11.24 & 11.26 & 11.08 & 11.15 & 11.19 \\
\hline T8 & $\begin{array}{l}50 \% \mathrm{~N}+\text { Reco. } \mathrm{P}+\mathrm{K}+\mathrm{S}+\mathrm{Zn}+\mathrm{B}+\mathbf{5 0 \%} \mathrm{N} \\
\text { through FYM +Azot }+\mathrm{PSB}\end{array}$ & 14.31 & 14.29 & 14.30 & 13.62 & 13.88 & 13.75 \\
\hline T9 & $\begin{array}{l}50 \% \mathrm{~N}+\text { Reco. } \mathrm{P}+\mathrm{K}+\mathrm{S}+\mathrm{Zn}+\mathrm{B}+\mathbf{5 0 \%} \mathrm{N} \\
\text { through VC + Azot + PSB }\end{array}$ & 13.08 & 13.44 & 13.26 & 12.98 & 13.30 & 13.14 \\
\hline T10 & $\begin{array}{l}\text { 75\% N+Reco.P +K }+\mathrm{S}+\mathrm{Zn}+\mathrm{B}+25 \% \mathrm{~N} \\
\text { through FYM +Azto +PSB }\end{array}$ & 11.62 & 11.68 & 11.65 & 11.32 & 11.55 & 11.44 \\
\hline T11 & $\begin{array}{l}75 \% \mathrm{~N}+\text { Reco. } \mathrm{P}+\mathrm{K}+\mathrm{S}, \mathrm{Zn}+\mathrm{B}+25 \% \mathrm{~N} \\
\text { through VC }+ \text { Azot }+\mathrm{PSB}\end{array}$ & 11.54 & 11.64 & 11.59 & 11.22 & 11.42 & 11.32 \\
\hline & \pm S.E. $(\mathrm{m})$ & 0.07 & 0.14 & 0.19 & 0.09 & 0.16 & 0.07 \\
\hline & C.D. $(P=0.05)$ & 0.010 & 0.042 & 0.037 & 0.27 & 0.49 & 0.22 \\
\hline
\end{tabular}

Table.3 Integrated effect of inorganic, organic and biofertilizers on soil reaction (1:2.5) soil after harvesting maize wheat cropping system

\begin{tabular}{|c|c|c|c|c|c|c|c|}
\hline \multirow[b]{2}{*}{ Sr.No } & \multirow[b]{2}{*}{ Treatments } & $I^{\text {st }}$ year & II $^{\text {nd }}$ year & Overall & $I^{\text {st }}$ year & II $^{\text {nd }}$ year & Overall \\
\hline & & \multicolumn{3}{|c|}{$(1: 2.5)$} & \multicolumn{3}{|c|}{$(1: 2.5)$} \\
\hline T1 & Control & 6.54 & 6.61 & 6.58 & 6.55 & 6.51 & 6.53 \\
\hline T2 & Recommended NPK & 6.59 & 6.58 & 6.59 & 6.53 & 6.49 & 6.51 \\
\hline T3 & Reco. NPK+ S + Zn + B & 6.56 & 6.50 & 6.53 & 6.58 & 6.48 & 6.53 \\
\hline T4 & $\begin{array}{l}50 \% \mathrm{~N}+\text { Reco. } P+\mathrm{K}+\mathrm{S}+\mathrm{Zn}+\mathrm{B}+50 \% \\
\mathrm{~N} \text { through FYM }\end{array}$ & 6.54 & 6.56 & 6.55 & 6.52 & 6.47 & 6.50 \\
\hline T5 & $\begin{array}{l}50 \% \mathrm{~N}+\text { Reco. } \mathrm{P}+\mathrm{K}+\mathrm{S}+\mathrm{Zn}+\mathrm{B}+\mathbf{5 0 \%} \\
\mathrm{N} \text { through VC }\end{array}$ & 6.42 & 6.33 & 6.38 & 6.48 & 6.47 & 6.48 \\
\hline T6 & $\begin{array}{l}75 \% \mathrm{~N}+\text { Reco. } \mathrm{P}+\mathrm{K}+\mathrm{S}+\mathrm{Zn}+\mathrm{B}+25 \% \mathrm{~N} \\
\text { through FYM }\end{array}$ & 6.46 & 6.33 & 6.40 & 6.47 & 6.45 & 6.46 \\
\hline T7 & $\begin{array}{l}75 \% \mathrm{~N}+\text { Reco. } \mathrm{P}+\mathrm{K}+\mathrm{S}+\mathrm{Zn}+\mathrm{B}+25 \% \mathrm{~N} \\
\text { through VC }\end{array}$ & 6.48 & 6.38 & 6.43 & 6.45 & 6.41 & 6.43 \\
\hline T8 & $\begin{array}{l}50 \% \mathrm{~N}+\text { Reco. } \mathrm{P}+\mathrm{K}+\mathrm{S}+\mathrm{Zn}+\mathrm{B}+\mathbf{5 0 \%} \mathrm{N} \\
\text { through FYM +Azot }+\mathrm{PSB}\end{array}$ & 6.50 & 6.44 & 6.47 & 6.42 & 6.48 & 6.45 \\
\hline T9 & $\begin{array}{l}50 \% \mathrm{~N}+\text { Reco. } \mathrm{P}+\mathrm{K}+\mathrm{S}+\mathrm{Zn}+\mathrm{B}+\mathbf{5 0 \%} \mathrm{N} \\
\text { through VC }+ \text { Azot }+\mathrm{PSB}\end{array}$ & 6.49 & 6.50 & 6.50 & 6.41 & 6.46 & 6.44 \\
\hline T10 & $\begin{array}{l}75 \% \mathrm{~N}+\text { Reco.P }+\mathrm{K}+\mathrm{S}+\mathrm{Zn}+\mathrm{B}+25 \% \mathrm{~N} \\
\text { through FYM +Azto +PSB }\end{array}$ & 6.42 & 6.39 & 6.41 & 6.39 & 6.44 & 6.42 \\
\hline T11 & $\begin{array}{l}75 \% \mathrm{~N}+\operatorname{Reco.} \mathrm{P}+\mathrm{K}+\mathrm{S}, \mathrm{Zn}+\mathrm{B}+25 \% \mathrm{~N} \\
\text { through VC }+ \text { Azot }+\mathrm{PSB}\end{array}$ & 6.22 & 6.42 & 6.32 & 6.43 & 6.39 & 6.41 \\
\hline & \pm S.E. $(\mathrm{m})$ & 0.02 & 0.03 & 0.05 & 0.02 & 0.01 & 0.02 \\
\hline & C.D. $(P=0.05)$ & 0.06 & 0.09 & 0.15 & 0.07 & 0.04 & 0.08 \\
\hline
\end{tabular}


Table.4 Integrated effect of inorganic, organic and biofertilizers on organic carbon $\left(\mathrm{g} \mathrm{kg}^{-1}\right)$ soil after harvesting maize wheat cropping system

\begin{tabular}{|c|c|c|c|c|c|c|c|}
\hline \multirow[b]{2}{*}{ Sr.No } & \multirow[b]{2}{*}{ Treatments } & $I^{\text {st }}$ year & II $^{\text {nd }}$ year & Overall & $I^{\text {st }}$ year & II $^{\text {nd }}$ year & Overall \\
\hline & & \multicolumn{3}{|c|}{$(\mathrm{g} \mathrm{kg}-1)$} & \multicolumn{3}{|c|}{$(\mathrm{g} \mathrm{kg}-1)$} \\
\hline T1 & Control & 2.22 & 2.28 & 2.25 & 2.23 & 2.26 & 2.26 \\
\hline $\mathbf{T} 2$ & Recommended NPK & 2.27 & 2.34 & 2.31 & 2.28 & 2.33 & 2.31 \\
\hline T3 & Reco. $\mathbf{N P K}+\mathbf{S}+\mathbf{Z n}+\mathbf{B}$ & 2.35 & 2.41 & 2.38 & 2.37 & 2.45 & 2.41 \\
\hline T4 & $\begin{array}{l}50 \% \mathrm{~N}+\text { Reco. } P+\mathrm{K}+\mathrm{S}+\mathrm{Zn}+\mathrm{B} \\
+\mathbf{5 0 \%} \mathrm{N} \text { through FYM }\end{array}$ & 3.02 & 3.44 & 3.23 & 3.09 & 3.48 & 3.29 \\
\hline T5 & $\begin{array}{l}50 \% \mathrm{~N}+\operatorname{Reco.} \mathrm{P}+\mathrm{K}+\mathrm{S}+\mathrm{Zn}+\mathrm{B} \\
+\mathbf{5 0 \%} \mathrm{N} \text { through VC }\end{array}$ & 2.82 & 3.23 & 3.03 & 3.04 & 3.06 & 3.05 \\
\hline T6 & $\begin{array}{l}75 \% \mathrm{~N}+\text { Reco. } \mathrm{P}+\mathrm{K}+\mathrm{S}+\mathrm{Zn}+ \\
\mathrm{B}+25 \% \mathrm{~N} \text { through FYM }\end{array}$ & 2.49 & 2.68 & 2.58 & 2.79 & 2.94 & 2.87 \\
\hline T7 & $\begin{array}{l}75 \% \mathrm{~N}+\text { Reco. } \mathrm{P}+\mathrm{K}+\mathrm{S}+\mathrm{Zn} \\
+\mathrm{B}+25 \% \mathrm{~N} \text { through } \mathrm{VC}\end{array}$ & 2.51 & 2.55 & 2.53 & 2.64 & 2.79 & 2.72 \\
\hline T8 & $\begin{array}{l}50 \% \text { N+ Reco. } \mathrm{P}+\mathrm{K}+\mathrm{S}+\mathrm{Zn}+\mathrm{B}+ \\
\mathbf{5 0 \%} \mathrm{N} \text { through FYM }+ \text { Azot }+\mathrm{PSB}\end{array}$ & 4.11 & 4.12 & 4.12 & 4.14 & 4.19 & 4.17 \\
\hline T9 & $\begin{array}{l}50 \% \mathrm{~N}+\text { Reco. } \mathrm{P}+\mathrm{K}+\mathrm{S}+\mathrm{Zn}+\mathrm{B}+ \\
\mathbf{5 0 \%} \mathrm{N} \text { through } \mathrm{VC}+\text { Azot }+\mathrm{PSB}\end{array}$ & 4.09 & 4.02 & 4.06 & 4.00 & 4.05 & 4.03 \\
\hline T10 & $\begin{array}{l}75 \% \mathrm{~N}+\mathrm{Reco.P}+\mathrm{K}+\mathrm{S}+\mathrm{Zn}+\mathrm{B}+ \\
25 \% \mathrm{~N} \text { through } \mathrm{FYM}+\mathrm{Azto}+\mathrm{PSB}\end{array}$ & 2.69 & 2.85 & 2.77 & 2.79 & 3.00 & 2.89 \\
\hline T11 & $\begin{array}{l}75 \% \mathrm{~N}+\text { Reco. } \mathrm{P}+\mathrm{K}+\mathrm{S}, \mathrm{Zn}+\mathrm{B}+ \\
25 \% \mathrm{~N} \text { through } \mathrm{VC}+\mathrm{Azot}+\mathrm{PSB}\end{array}$ & 2.66 & 2.86 & 2.75 & 2.67 & 2.69 & 2.68 \\
\hline & \pm S.E. $(\mathrm{m})$ & 0.03 & 0.13 & 0.16 & 0.07 & 0.06 & 0.03 \\
\hline & C.D. $(P=0.05)$ & 0.11 & 0.40 & 0.53 & 0.20 & 0.20 & 0.11 \\
\hline
\end{tabular}

These observation corroborate the results obtained by Rehman et al., 2017 and Thangasamy et al., 2017.

The highest WHC after harvesting of maize and wheat was found in $\mathrm{T}_{8}$ (14.31) to lowest in $T_{1}(11.25)$ and $T_{8}(13.62)$ to lowest in $T_{1}$ (11.11)can be due to addition of organic, inorganics and biofertilizers which catalyzed to enhanced organic carbon, and thereby increasing water holding capacity these findings are corroborate the observation of Sharma et al., (2017).

The highest $\mathrm{pH}$ of soil after harvesting of maize and wheat crop was noticed in $\mathrm{T}_{2}$ (6.59) to lowest $\mathrm{T}_{11}(6.22)$ and $\mathrm{T}_{3}(6.58)$ to lowest in $\mathrm{T}_{10}$ (6.39) the significant improvements in soil $\mathrm{pH}$ under treatments, might be due to integrated application of organic, inorganic and biofertilizers which brought significant enhancement in soil $\mathrm{pH}$ under aforesaid treatments. were These observation are in agreement with the results of Mishra et al., (2008), Chesti et al., (2013) and Rehman et al., (2017).

The highest value of organic carbon after harvesting of maize and wheat crop was recorded in overall organic carbon found highest in $\mathrm{T}_{8}$ (4.12) to lowest in $\mathrm{T}_{1}(2.25)$ and highest in $\mathrm{T}_{8}$ (4.17) to lowest in $\mathrm{T}_{1}$ (2.26). The highest value of organic carbon in $\mathrm{T}_{8}(4.12)$ may be due to integrated uses of organic, inorganic and biofertilizers which released its higher uptake whereas lowest 
value of organic carbon may be due its scanty retention. The decreasing pattern of overall organic carbon under different treatments might be due to its slow release pattern responsible for decreasing trend. These observations are supported by Kumari et al., (2011) and Lakaria et al., (2012).

It is concluded that over two year of maizewheat cropping system respect the treatments receiving integrated use of organic, inorganic and biofertilizers improved the soil status of soil properties viz, $\mathrm{pH}$, Organic carbon, water holding capacity, bulk density improved significantly under treatments received integrated nutrient application.

\section{References}

Chesti, M.H., Kohli, A. and Sharma A.K. 2013. Effect of integrated nutrient management on yield of and nutrient uptake by wheat (Triticum aestivum) and soil properties under intermediate zone of Jammu and Kashmir. Journal of the Indian Society of Soil Science. 61 (1):1-6.

Chesnin, L., and Yein, C.H. 1951. Turbidimetric determination of available sulphur. Soil Science of America Proceedings 15, 149-151.

Chhonkar, P.K., Bhadraray, S., Patra, A.K. and Purakayastha, T.J. 2002. Practical Manual on Soil Biology and Biochemistry. Indian Agricultural Research Institute, New Delhi-110 012. Pp. 57.

Ghuman, B.S., and Sur, H.S. 2006. Effect of manuring on soil properties and yield of rainfed wheat. Journal of the Indian Society of Soil Science. 54(1): 6-11

Jockson, M.L. 1973. Soil Chemical Analysis, Prentice hall of India Pvt. Limited. Lakharia, B.L., Patne,
Kumar, M., Jha, P. and Biswas, A.K. 2012. Soil organic carbon pools and indices under different land use systems in vertisols of central India. Journal of the Indian Society of Soil Science. 65: 125-131.

Lindsay, W.L., and Norwell, W.A. 1978. Development of DTPA soil test for zinc, iron, manganese and copper. Soil Science Society of America Journal 42: 421-428.

Olsen, S.R., Cole, C.V., Watanable, F.S. and Dean, L.A. 1994 Extermination of available phosphorus in soils by extraction with sodium bicarbonate. USDA Circular 939.

Mishra, B., Sharma, A., Singh, S.K., Singh, Prasad, J. and Singh, B.P. 2008. Influence of continuous application of amendments to maize-wheat cropping system on dynamics of soil microbial biomass in alfisol of Jharkhand. Journal of the Indian Society of Soil Science. 56 (1):71-75.

Olsen, S.R., Cole, C.V., Watanable, F.S. and Dean, L.A. 1994 Extermination of available phosphorus in soils by extraction with sodium bicarbonate. USDA Circular 939.

Page, A.L. Miller, R.H. and Keeny, D.R. (eds.) 1982. Agronomy Monograph 9, ASA/SSSA Publication, Madison, Wisconsin

Piper, C.S., 1966. Soil and plant Analysis. Hans Publisher. Bombay, India.

Rehman, N.Z., Ram, D., Wani, J.A. and Maqbool, M. 2017. Characterization, classification and evaluation of cultivated soils under different top sequences in Pulwama district of Kashmir valley. Journal of the Indian Society of Soil Science. 65 (3):239-247.

Sharma, M.P., Sharma, Mondal, A.K., Bhoye, R. C., Samantha A., Rai1, A.P., Arya1, V.M., and Sharma, 
K.R.2017. Soil Characterization of Advance Research Centre for Rain Fed Agriculture Farm of Sher-eKashmir University of Agricultural Sciences and Technology RakhDhinsar, Jammu- (J\&K). International Journal of Plant \& Soil Science. 20(1): 1-7, 2017

Subbiah, B.V., and Asija, G. L. 1956. A rapid procedure for the determination of available nitrogen in soils. Current Sciences, 25: 259-68.

Thangasamy, A., Singh, D., Dwivedi, B.S.,
Chakraborty, D., Tomar, R.K. and Meena, M.C. 2017. Soil organic carbon, hydraulic properties and yield of maize and wheat under long term fertilization in an inceptisol. Journal of the Indian Society of Soil Science.65 (2):189-198.

Williams, C.H., and Heidel, H. 1952. Soil analysis methods as used in Iowa State College, Soil Testing Laboratory, Iowa State College Bull. 57: 1-131.

\section{How to cite this article:}

Bhoye Ranjanabai Chhagan, M.P. Sharma, K.R. Sharma, Abhijit Samanta, Owais Ali Wani, Dileep Kachroo, Manish Kumar, V.K. Razdan, Vikas Sharma, A.K. Mondal and Arya, V.M. 2019. Effect of Organic, Inorganic and Bio fertilizers on Soil Physicochemical Properties in Rainfed Maize-wheat Cropping System of Jammu. Int.J.Curr.Microbiol.App.Sci. 8(04): 25392545. doi: https://doi.org/10.20546/ijcmas.2019.804.296 